\title{
The search for organic compounds in the atmosphere and in sediments of an ancient martian lakebed
}

\author{
PAUL MAHAFFY ${ }^{1}$ \\ 'NASA Goddard Space Flight Center, Greenbelt, MD 20771 \\ USA Paul.R.Mahaffy@nasa.gov
}

The Curiosity rover on Mars has now been sampling rocks, scooped soils, and the atmosphere for more than 7 years in an ancient martian impact crater that once contained a long-lived lake more than 3 billion years ago. The goal of the Mars Science Laboratory (MSL) mission is the exploration of the habitability of Gale crater. To date, the Sample Analysis at Mars (SAM) instrument suite in the interior of Curiosity rover has conducted geochemical investigations that include detection of methane plumes, the seasonal variation of a lower abundance methane background, and detection of a variety of organic compounds extracted from soils and rocks. Other SAM investigations that provide information on the ancient environment and its transformation over time include: abundance and isotopic composition of atmospheric gases and those thermally evolved from solid samples; rock formation ages from K/Ar measurements; cosmic radiation exposure ages from noble gases released from minerals; and evolved gas signatures from clays, oxychlorine, sulfur, and nitrogen compounds. These measurements of volatiles are complemented by elemental abundances secured from the alpha-particle x-ray spectrometer and the laser induced breakdown spectrometer on the rover and mineralogical data from an x-ray diffractometer. Cameras, environmental monitors, and a neutron spectrometer round out the MSL payload.

The first in situ detection of organic compounds from the surface of Mars using GCMS was of chlorobenzene and three chlorinated alkanes from the Cumberland mudstone. Sulfur compounds such as thiophene, methyl and di-methyl thiophene, dimethylsulfide, and methanethiol were also identified, as well as decane, dodecane, and fragments of other aromatic and alkyl compounds. Chemical derivatization experiments that expand the breadth of the organics analysis have been initiated and are planned to continue as the rover traverses through clay and sulfur rich regions where organic compounds may be preserved. These investigations of organic compounds on Mars and mechanism responsible for their preservation or degradation in rocks pave the way for a more direct search for extinct or extant life on this planet.

The MSL/SAM investigation is supported by an international team of scientists and operations personnel. 